\title{
Presenting for service
}

Oncogenic tumour-associated proteins are often expressed intracellularly and so are not accessible to classical antibody-based therapy. Taking advantage of the fact that intracellular proteins can be degraded in the proteasome, processed and presented on the cell surface as T cell epitopes, a recent study in Science Translational Medicine has identified a monoclonal antibody that recognized a cell surface epitope of the previously intracellular form of the Wilms' tumour 1 (WT1) oncoprotein. This antibody bound to a range of cancer cell lines and cleared established and disseminated leukaemias in animal models.

The WT1 oncoprotein is an intracellular oncogenic transcription factor that is overexpressed in many leukaemias and solid cancers but rarely expressed in normal cells. Previous studies have shown that a peptide (RMFPNAPYL) derived from this protein is processed and presented by major histocompatibility complex (MHC) class I human leukocyte antigen (HLA)-A0201 molecules and induces peptidespecific cytotoxic CD8 T cells that are capable of killing tumour cells in vitro and in vivo.

Therefore the authors used phage display to find antibodies that would recognize the WT1 peptide epitope. Phage clones that were selective for the WT1 peptide-HLA-A0201 complex were made into full-length human monoclonal antibodies. One antibody - termed ESK1 that showed the strongest binding to the WT1 epitope was used for further studies. ESK1 recognized the WT1 epitope presented on the surface of several cancer cell lines, including

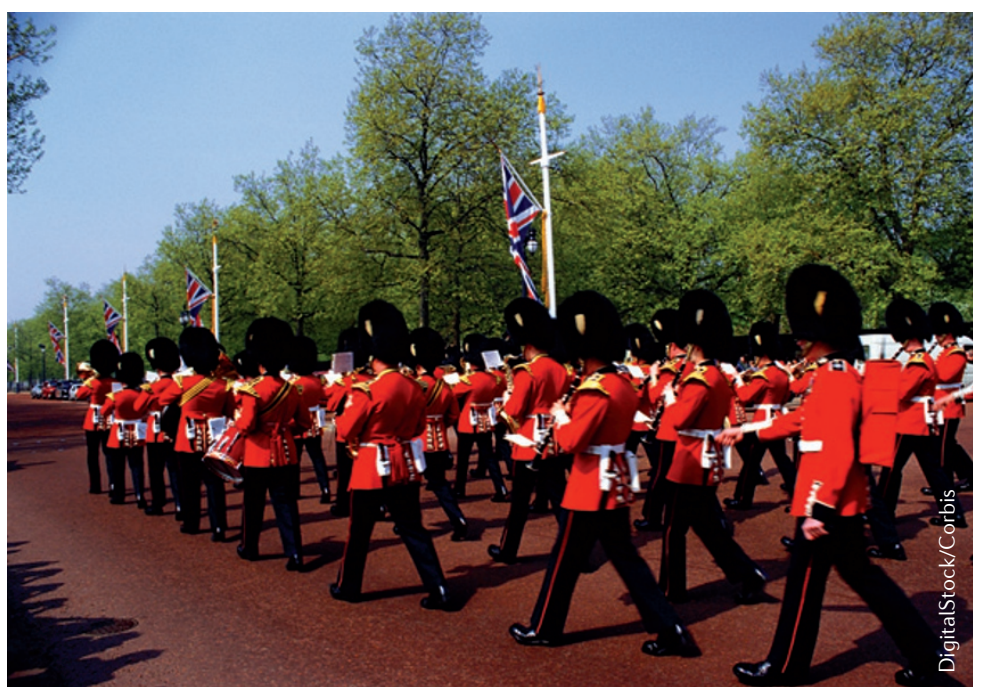

human mesothelioma cells, human haematopoietic cells as well as fresh leukaemia cells, but did not bind to several healthy cell lines.

Next, the authors determined that ESK1 was able to induce antibodydependent cell-mediated cytotoxicity (ADCC) in a panel of cancer cell lines. The antibody had no effects in assays of complement-mediated cytotoxicity, antibody-dependent cellular phagocytosis or direct killing via apoptosis.

Next, the efficacy of ESK1 was tested in a xenograft model of disseminated $B c r-A b l$-positive acute leukaemia. Two doses of ESK1, together with human natural killer cells, B cells and monocytes (that is, $\mathrm{CD}^{-} \mathrm{CD} 34^{-}$cells, as these were not present in the mouse model), almost ablated the leukaemia. In addition, two doses of ESK1 without human immune cells also markedly reduced the tumour burden but the mice relapsed more quickly than mice that were given ESK1 supplemented with human immune cells, which suggests that ESK1-mediated ADCC probably has an important role in the longterm elimination of tumour cells. Indeed, a variant of ESK1 that was engineered to have improved ADCC activity showed better therapeutic effects in this mouse model. ESK1 also suppressed tumour growth in a second mouse model of disseminated leukaemia (BA25 acute lymphocytic leukaemia).

So this study complements other studies that have demonstrated that it is possible to produce antibodies against other intracellular oncogenic proteins, and opens the door for therapies against the many tumours that express the WT1 oncogenic protein.

Charlotte Harrison

ORIGINAL RESEARCH PAPER Dao, T. et al. Targeting the intracellular WT1 oncogene product with a therapeutic human antibody. Sci. Transl. Med. 5, 176ra33 (2013) 